\title{
Muon spin rotation and neutron scattering study of the noncentrosymmetric tetragonal compound $\mathrm{CeAuAl}_{3}$
}

\author{
D. T. Adroja ${ }^{1,2,{ }^{*}}$ C. de la Fuente, ${ }^{3}$ A. Fraile,,${ }^{1,3}$ A. D. Hillier, ${ }^{1}$ A. Daoud-Aladine, ${ }^{1}$ W. Kockelmann, ${ }^{1}$ J. W. Taylor, ${ }^{1}$ \\ M. M. Koza, ${ }^{4}$ E. Burzurí, ${ }^{3}$ F. Luis,${ }^{3}$ J. I. Arnaudas, ${ }^{3}$ and A. del Moral ${ }^{3}$ \\ ${ }_{1}^{1}$ ISIS Facility, Rutherford Appleton Laboratory, Chilton, Didcot, Oxon OX11 0QX, United Kingdom \\ ${ }^{2}$ Highly Correlated Electron Group, Physics Department, University of Johannesburg, P.O. Box 524, Auckland Park 2006, South Africa \\ ${ }^{3}$ Depto. Física Materia Condensada and Instituto de Ciencia de Materiales de Aragón, Universidad de Zaragoza and Consejo Superior de \\ Investigaciones Científicas, 50071, Zaragoza, Spain \\ ${ }^{4}$ Institut Laue-Langevin, BP 156, F-38042 Grenoble Cedex 9, France
}

(Received 30 December 2014; revised manuscript received 30 March 2015; published 24 April 2015)

\begin{abstract}
We have investigated the noncentrosymmetric tetragonal heavy fermion compound $\mathrm{CeAuAl}_{3}$ using muon spin rotation $(\mu \mathrm{SR})$, neutron diffraction (ND), and inelastic neutron scattering (INS) measurements. We have also revisited the magnetic, transport, and thermal properties. The magnetic susceptibility reveals an antiferromagnetic transition at $1.1 \mathrm{~K}$ with, possibly, another magnetic transition near $0.18 \mathrm{~K}$. The heat capacity shows a sharp $\lambda$-type anomaly at $1.1 \mathrm{~K}$ in zero field, which broadens and moves to a higher temperature in an applied magnetic field. Our zero-field $\mu$ SR and ND measurements confirm the existence of a long-range magnetic ground state below $1.2 \mathrm{~K}$. Further, the ND study reveals an incommensurate magnetic order with a magnetic propagation vector $\mathbf{k}=(0,0,0.52(1))$ and a spiral structure of Ce moments coupled ferromagnetically within the $a b$ plane. Our INS study reveals the presence of two well-defined crystal electric field (CEF) excitations at 5.1 and $24.6 \mathrm{meV}$ in the paramagnetic phase of $\mathrm{CeAuAl}_{3}$ that can be explained on the basis of the CEF theory and the Kramer's theorem for a $\mathrm{Ce}$ ion having a $4 f^{1}$ electronic state. Furthermore, low energy quasielastic excitations show a Gaussian line shape below $30 \mathrm{~K}$ compared to a Lorentzian line shape above $30 \mathrm{~K}$, indicating a slowdown of spin fluctuations below $30 \mathrm{~K}$. We have estimated a Kondo temperature of $T_{\mathrm{K}}=3.5 \mathrm{~K}$ from the quasielastic linewidth, which is in good agreement with that estimated from the heat capacity. This study also indicates the absence of any CEF-phonon coupling unlike that observed in isostructural $\mathrm{CeCuAl}_{3}$ The $\mathrm{CEF}$ parameters, energy level scheme, and their wave functions obtained from the analysis of INS data explain satisfactorily the single crystal susceptibility in the presence of two-ion anisotropic exchange interaction in $\mathrm{CeAuAl}_{3}$.
\end{abstract}

DOI: 10.1103/PhysRevB.91.134425

PACS number(s): 71.27.+a, 75.30.Mb, 71.70.Ch, 78.70.Nx

\section{INTRODUCTION}

The antiferromagnetic (AFM) $s-f$ exchange coupling, $J_{\text {sf }}$, between conduction and localized spins in heavy fermion (HF) rare-earth systems is responsible for two competing effects: the screening of the onsite localized moments due to the Kondo effect and the intrasite Ruderman-Kittel-KasuyaYosida (RKKY) interaction among the magnetic impurities that may induce a long-range magnetic ordering. The Doniach phase diagram describes this competition [1]. First, the Néel temperature $T_{\mathrm{N}}$ rises on increasing the absolute value of the exchange interaction constant $J_{\text {sf }}$ or hybridization strength, $V_{\text {sf }}$, between conduction and localized electron states. Then, $T_{\mathrm{N}}$ passes through a maximum with further increases in $J_{\mathrm{sf}}$ (or $V_{\text {sf }}$ ), and finally, it tends to decrease to zero at the "quantum critical point" (QCP). Such a decrease of $T_{\mathrm{N}}$ down to the QCP has been observed in many Ce-based HF compounds [2]. At present, various theoretical scenarios exist to explain the observed behavior of the systems close to QCP, and they are classified into two major categories: (1) local QCP, where $T_{\mathrm{K}} \rightarrow 0$ at QCP [2-4] and (2) spin density wave scenario, where $T_{\mathrm{K}}$ remains finite at $\mathrm{QCP}[5,6]$. Above the $\mathrm{QCP}$, a very strong $\mathrm{HF}$ character will eventually reduce the $T_{\mathrm{K}}$, and these systems exhibit non-Fermi-liquid (NFL) properties [7-9].

\footnotetext{
*Corresponding author: devashibhai.adroja@stfc.ac.uk
}

Cerium-based HF intermetallic compounds with the general formula $\mathrm{CeTX}_{3}$ (1-1-3 stoichiometry, with $T=$ transition metals and $X=\mathrm{Si}, \mathrm{Ge}$, and $\mathrm{Al}$ ), have recently attracted considerable experimental and theoretical interest [10-15]. The reason is due to the discovery of many novel ground state properties in the tetragonal noncentrosymmetric crystal structure, such as unconventional superconductivity in $\mathrm{CeTSi}_{3}$, $T=\mathrm{Ir}$ and $\mathrm{Rh}$, and $\mathrm{CeCoGe}_{3}$, at around QCP under pressure [16-19]. The tetragonal $\mathrm{CeAuAl}_{3}$ belongs to the above class of compounds and could have a similar strength of Kondo and RKKY interactions. The thermal and transport properties of $\mathrm{CeAuAl}_{3}$ at low temperatures suggest the presence of strongly correlated electrons in a "magnetically ordered" phase [20-22]. Furthermore, $\mathrm{CeAuAl}_{3}$ shows a large electronic coefficient $\left(\gamma_{\text {elec }}\right)$ at zero field $(\mathrm{ZF}), \approx 227 \mathrm{~mJ} / \mathrm{mole}^{2} \mathrm{~K}^{2}$, and a large coefficient of the quadratic term in the magnetoresistivity, $\approx 4.84 \mu \Omega \mathrm{cm} / \mathrm{K}^{2}$. $\mathrm{CeAuAl}_{3}$ has been reported to order antiferromagnetically at $\sim 1.3 \mathrm{~K}[20,21]$. The heat capacity, magnetic susceptibility, and resistivity measurements existing in the literature clearly show the influence of the crystal electric field (CEF) at around 10-50 K. The nuclear magnetic resonance (NMR) study of ${ }^{27} \mathrm{Al}$ in $\mathrm{CeAuAl}_{3}$ shows that the $\mathrm{Ce}$ magnetic moments are ordered, and their magnitude reduced by $\sim 25 \%$ at $0.50 \mathrm{~K}$, most likely due to Kondo screening [22].

Furthermore, those systems with strongly correlated electrons can show spin, charge, and lattice degrees of freedom that sustain low energy magnetic or CEF excitations very 
similar in energy scales of lattice vibrations (i.e., phonons). In most of these systems, these excitations remain decoupled; therefore, they can be studied independently. Particularly interesting are those systems where strong CEF-phonon (or spin-phonon) coupling exists. Recently, we have investigated the noncentrosymmetric tetragonal $\mathrm{CeCuAl}_{3}$ compound using inelastic neutron scattering (INS) and found the presence of three excitations in the paramagnetic phase at $1.25,10$, and $20 \mathrm{meV}$ [23]. Based on Kramer's theorem, we cannot expect more than two CEF excitations for $\mathrm{Ce}^{3+}\left(4 f^{1}\right)$ in the paramagnetic phase. The observed three $\mathrm{CEF}$ excitations in $\mathrm{CeCuAl}_{3}$ have been explained based on the CEF-phonon coupling model (called magnetoelastic coupling) [23]. In order to investigate whether or not the CEF-phonon coupling is also present in other members of the noncentrosymmetric compounds with the general formula $\mathrm{CeTX}_{3}$, we are currently investigating several compounds of this family using INS [10$12,23]$. In the present paper, we have investigated the tetragonal $\mathrm{CeAuAl}_{3}$ compound using various bulk characterization techniques, muon spin rotation $(\mu \mathrm{SR})$, as well as using neutron scattering (both elastic and inelastic). Our study reveals the presence of two CEF excitations in the paramagnetic phase indicating the absence of CEF-phonon coupling in $\mathrm{CeAuAl}_{3}$. Further, neutron diffraction (ND) reveals an incommensurate magnetic structure with a magnetic propagation vector $\mathbf{k}=$ $(0,0,0.52(1))$ and a spiral structure of Ce moments coupled ferromagnetically within the $a b$ plane.

\section{EXPERIMENTAL DETAILS}

Polycrystalline samples of $\mathrm{CeAuAl}_{3}$ and $\mathrm{LaAuAl}_{3}$ were prepared by the standard arc melting method starting with a stoichiometric mixture of the high purity elements (Ce, La: 99.9\%, Cu: 99.99\%, Al: 99.999\%). The as-cast samples were annealed for a week at $850^{\circ} \mathrm{C}$ under high vacuum to improve the phase formation. The phase purity of $\mathrm{LaAuAl}_{3}$ was checked using $\mathrm{X}$-ray diffraction (XRD) at room temperature and of $\mathrm{CeAuAl}_{3}$ using $\mathrm{ND}$ at $9 \mathrm{~K}$. The $\mathrm{XRD}$ study was carried out at room temperature by using a Rigaku/Max system model Rotaflex RU-300 with a graphite monochromator.

The heat capacity $\left(C_{p}\right)$, magnetization, and electrical resistivity $(\rho)$ have been measured using a commercial Physical Property Measurement System (PPMS) from Quantum Design. The $C_{p}$ was measured by using an adiabatic heat pulse type calorimeter between 0.350 and $300 \mathrm{~K}$. The magnetization and dc susceptibility measurements were carried out using a superconducting quantum interference device (SQUID) magnetometer and a vibrating sample magnetometer, both from Quantum Design from 1.8 to $50 \mathrm{~K}$. Finally, the electrical resistivity was measured, from 0.350 to $300 \mathrm{~K}$, by means of a standard four-probe technique with the leads attached to the sample using silver epoxy paint. The measurements were taken with samples mounted on a superconducting coil with an applied magnetic field up to $9 \mathrm{~T}$. We have used a homemade mutual inductance thermally anchored to the mixing chamber of a ${ }^{3} \mathrm{He}-{ }^{4} \mathrm{He}$ dilution refrigerator, which enables measurements to be performed from $0.09 \mathrm{~K}$ up to $3.5 \mathrm{~K}$ in the frequency range between 333 and $13.3 \mathrm{kHz}$ and $\left(\mu_{0} \mathrm{H}\right)_{\max } \approx 10^{-3} \mathrm{~T}$. The sample was fixed to a sample holder centered inside the secondary coil of the susceptometer with
Apiezon $\mathrm{N}$ grease. As-measured values were calibrated by using dc susceptibility values because the out-of-phase signal obtained from the lock-in amplifier was below sensitivity limits.

The $\mu \mathrm{SR}$ experiments were performed on the MuSR spectrometer in the longitudinal geometry configuration at the ISIS Facility, United Kingdom, and the details of the experimental technique can be found in Ref. [10].

Neutron diffraction measurements were carried out using the time-of-flight (TOF) General Materials (GEM) diffractometer at the ISIS Facility. The powdered sample of $\mathrm{CeAuAl}_{3}$ was inserted into a copper can with a diameter of $6 \mathrm{~mm}$ and placed inside a standard Oxford He-3 system with a base temperature of $0.3 \mathrm{~K}$. The measuring time was $6 \mathrm{~h}$ at each temperature, and data were collected at $0.3,0.75$, and $2 \mathrm{~K}$. Measurements at $9 \mathrm{~K}$ were also performed with the sample filled into a vanadium can and mounted inside a He-4 cryostat to characterize the sample quality. Each of the six detector banks of GEM provides a diffraction pattern for each measurement. The data from the six arrays are used in a multipattern Rietveld analysis. The INS measurements were carried out on the TOF spectrometers: (1) MARI at the ISIS Facility from $4.5 \mathrm{~K}$ to $250 \mathrm{~K}$, and (2) IN6, at Institut Laue-Langevin (ILL), Grenoble, France, for the low energy and high-resolution measurements. The measurements on MARI were performed with an incident neutron energy $E_{i}=35 \mathrm{meV}$ with elastic resolution of $0.6 \mathrm{meV}$ and on IN6 with $E_{i}=3.1 \mathrm{meV}$ with a resolution of $0.08 \mathrm{meV}$ at the elastic position. The powder samples were mounted in a thin cylindrical aluminum foil sample holder. The samples were cooled down to $4.5 \mathrm{~K}$ using a closed cycle refrigerator under He-exchange gas to thermalize the sample. On IN6, we used a standard orange cryostat down to $2 \mathrm{~K}$. In order to correct variations of the detector efficiency across the detector banks, the neutron counts from the standard vanadium sample were used to normalize the data. Further, the MARI data were presented in absolute units, millibarn, milli-electron volt, steradian, and formula units, by using the absolute normalization obtained from the standard vanadium sample measured in identical conditions.

\section{RESULTS}

\section{A. Structural characterization}

First, we discuss the structural characterization of $\mathrm{CeAuAl}_{3}$ using $\mathrm{ND}$ at $9 \mathrm{~K}$ and of $\mathrm{LaAuAl}_{3}$ using XRD at $300 \mathrm{~K}$. The analysis of ND and XRD data reveals that both samples were single phase and crystallize in the $\mathrm{BaNiSn}_{3}$-type tetragonal structure [24]. Figure 1 shows one of the six ND patterns of $\mathrm{CeAuAl}_{3}$ obtained on GEM at $9 \mathrm{~K}$ with the Rietveld refinement fit based on a tetragonal $\mathrm{BaNiSn}_{3}$-type structure (space group $I 4 m m$, No. 107). During this process, the site occupancies of the $\mathrm{Au}$ and $\mathrm{Al}$ atoms were varied, while keeping the site occupancy of the Ce atom fixed to $100 \%$. As the weighted profile reliability factor did not improve with Al site occupancies as variables, we kept Al occupancies fixed to $100 \%$ in the final refinement. The nearest neighbor distances for $\mathrm{CeAuAl}_{3}$ are (in angstroms) 4.3960 for $\mathrm{Ce}-\mathrm{Ce}$, 3.4226 for $\mathrm{Ce}-\mathrm{Au}, 3.2104$ for $\mathrm{Ce}-\mathrm{Al}(1), 3.2498$ for $\mathrm{Ce}-\mathrm{Al}(2)$, 


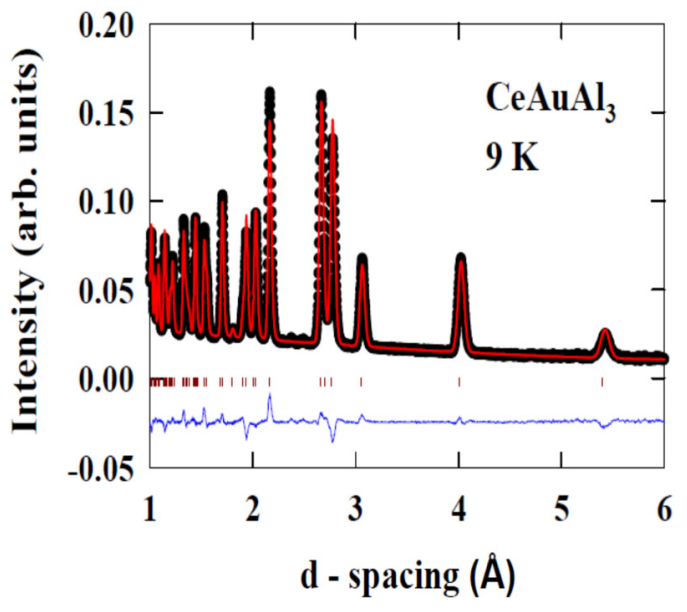

FIG. 1. (Color online) Rietveld refinement of the neutron powder diffraction pattern of $\mathrm{CeAuAl}_{3}$ in a vanadium can at $9 \mathrm{~K}$. The data are shown as closed circles, and the result of the refinement as a solid (red) line. The tick marks indicate the positions of nuclear Bragg peaks. The difference curve between the experimental data and the fitted pattern is shown at the bottom.

and 2.3900 for $\mathrm{Au}-\mathrm{Al}(1)$. The distance between $\mathrm{Au}-\mathrm{Al}(1)$ is the shortest among these distances, with four $\mathrm{Al}(1)$ atoms being at these distances from Au. Furthermore, the four $\mathrm{Al}(1)$ atoms have the shortest distances from the $\mathrm{Ce}$ atoms, which emphasizes the importance of Ce- $4 f$ and $\mathrm{Al}-4 p$ hybridization on the physical properties of $\mathrm{CeAuAl}_{3}$ and could explain why the superconducting properties of the Si-based compounds, $\mathrm{CeTSi}_{3}(T=\mathrm{Co}, \mathrm{Rh}$, and $\mathrm{Ir})$, are different from those of the Al-based compounds. A summary of the structural results for $(\mathrm{Ce} / \mathrm{La}) \mathrm{AuAl}_{3}$ is given in Table $\mathrm{I}$. Both compounds are in agreement with the published data, [20-22,24] and are quite similar from a structural point of view. Further, note that the lattice parameters of $\mathrm{CeAuAl}_{3}$ at $300 \mathrm{mK}$ are $a=4.3105(2) \AA$ and $c=10.7965(3) \AA(c / a=2.5047)$ and at $9 \mathrm{~K} \mathrm{a}=4.3172(2) \AA$ and $c=10.8090(3) \AA(c / a=2.5037)$. This shows a very small change in $c / a$ ratio above and below $T_{\mathrm{N}}$.

TABLE I. Rietveld-refined values of lattice parameters and position parameters $(z)$ from the ND and XRD patterns of $\mathrm{CeAuAl}_{3}$ and $\mathrm{LaAuAl}_{3}$ polycrystalline samples (space group $\mathrm{I} 4 \mathrm{~mm}$, No. 107), respectively. The $z$ parameter of $\mathrm{Ce} / \mathrm{La}$ was fixed at 0.0 taking account of the arbitrary origin for the noncentrosymmetric space group. Site occupancies are in good agreement with the (1-1-3) stoichiometry of the studied samples with a disorder between $\mathrm{Au}$ and $\mathrm{Al}<2 \%$.

\begin{tabular}{|c|c|c|c|c|}
\hline & \multirow{2}{*}{\multicolumn{2}{|c|}{$\begin{array}{c}\mathrm{CeAuAl}_{3} \text { at } 9 \mathrm{~K} \\
a=4.3172(2) \AA \\
c=10.8090(3) \AA \\
\text { Wyckoff sites }\end{array}$}} & \multirow{2}{*}{\multicolumn{2}{|c|}{$\begin{array}{c}\mathrm{LaAuAl}_{3} \text { at } 300 \mathrm{~K} \\
a=4.3660(2) \AA \\
c=\operatorname{rec} 10.8445(3) \AA \\
\text { Wyckoff sites }\end{array}$}} \\
\hline & & & & \\
\hline & $2 a$ & $4 b$ & $2 a$ & $4 b$ \\
\hline $\mathrm{Ce} / \mathrm{La}$ & $(0.0)$ & - & $(0.0)$ & - \\
\hline $\mathrm{Au}$ & $0.631(1)$ & - & $0.671(1)$ & - \\
\hline $\operatorname{Al}(1)$ & $0.407(2)$ & - & $0.424(2)$ & - \\
\hline $\operatorname{Al}(2)$ & - & $0.258(1)$ & - & $0.251(1)$ \\
\hline
\end{tabular}

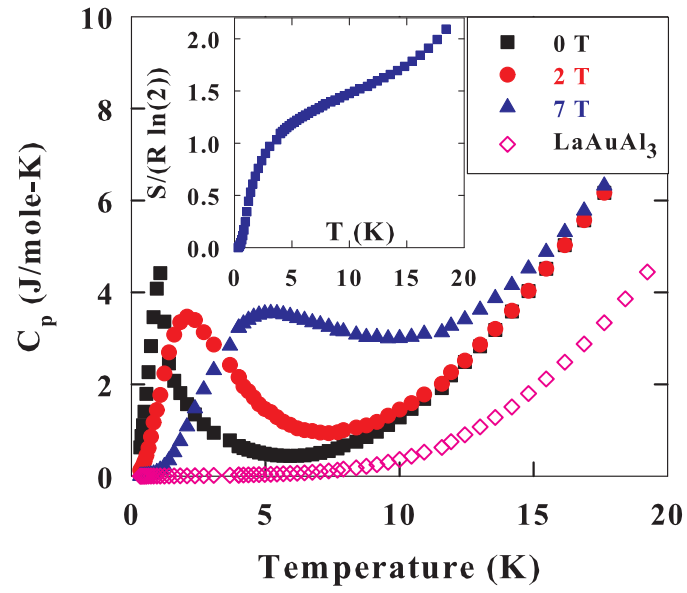

FIG. 2. (Color online) Thermal dependence of the heat capacity measured under different applied magnetic fields: $0 \mathrm{~T}(\boldsymbol{\square}), 2 \mathrm{~T}(\bullet)$, and $7 \mathrm{~T}(\boldsymbol{\Delta})$ for $\mathrm{CeAuAl}_{3}$. The $\mathrm{LaAuAl}_{3} \mathrm{ZF} C_{p}$ is also shown $(\diamond)$. The inset shows the temperature dependence of the magnetic entropy at $0 \mathrm{~T}$.

\section{B. Heat capacity}

Figure 2 shows the $C_{p}$ data of $\mathrm{CeAuAl}_{3}$ obtained under an applied magnetic field up to $7 \mathrm{~T}$. Our results of $C_{p}$ exhibited the $\lambda$-type anomaly at $T_{\mathrm{N}}=1.1(1) \mathrm{K}$ at $\mathrm{ZF}$, close to the values previously reported in the literature [20,21]. This anomaly shifts to high temperatures on increasing the applied magnetic field (at $7 \mathrm{~T}$ it shifts to $5 \mathrm{~K}$ ), and it becomes round and broad. There is no Schottky anomaly up to $10 \mathrm{~K}$ in the $\mathrm{ZF}$ data, which indicates that the CEF levels are higher than $10 \mathrm{~K}$. This is in agreement with our INS study discussed in Sec. III G. Further, the $C_{p}$ measurement of $\mathrm{LaAuAl}_{3}$ at $\mathrm{ZF}$ is shown in Fig. 2 exhibiting very low values at low temperature, as it can be expected. The linear $\mathrm{T}$ contribution due to the conduction electrons, $\gamma_{\text {elec }}$ is $3.24 \mathrm{~mJ} / \mathrm{mole}^{2} \mathrm{~K}^{2}$ and the $\mathrm{T}^{3}$-phonon-lattice contribution is $\sim 0.166 \mathrm{~mJ} / \mathrm{mole}-\mathrm{K}^{4}$. The calculated Debye temperature [25] is $227 \mathrm{~K}$. These results seem to be in good agreement with those values existing in the literature [20,21]. Figure 3 shows the electronic contribution to $C_{p}$ for $\mathrm{CeAuAl}_{3}$ at different applied magnetic fields. We can see how the

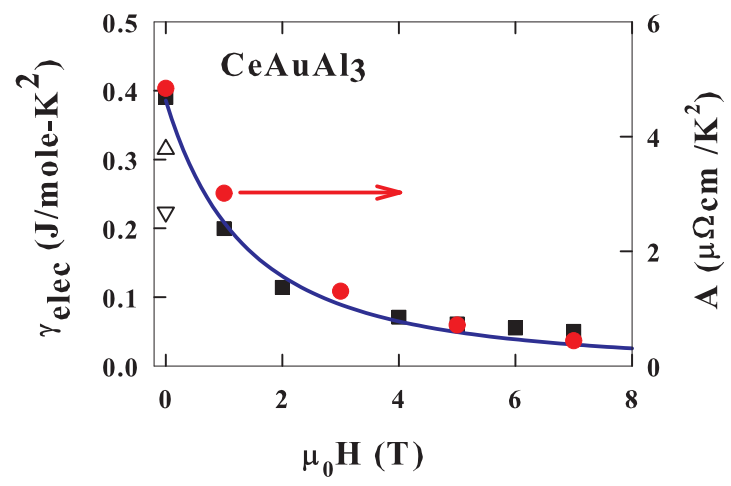

FIG. 3. (Color online) (Left) The magnetic field dependence of the electronic contribution $\gamma_{\text {elec }}$ to $C_{p}$ ( $\mathbf{\square}$ from 0 to $7 \mathrm{~T}, \Delta$ from Ref. [20], and $\Delta$ from Ref. [21]); (right) The $T^{2}$ coefficient (A) (•) of the resistivity at different magnetic fields. The solid line shows the fit (see text) to the $\gamma_{\text {elec }}(H)$ using Eq. (1). 
magnetic field suppresses the enhancement of $\gamma_{\text {elec., }}$ especially above $1 \mathrm{~T}$. This breaking of the Kondo effect could be due to the reduction of density of states (DOS) at Fermi level $\left(E_{\mathrm{F}}\right)$ induced by the magnetic field [26]. On the other hand, $C_{p}$ results allow us to estimate the Kondo temperature $T_{\mathrm{K}}$ in $\mathrm{CeAuAl}_{3}$. The inset of Fig. 2 shows the magnetic entropy in our system, and it is obtained from the experimental $C_{p}$ as, $S(T)=\int\left(C_{p}-C_{L}\right) d T / T$, where $C_{L}$ is the $C_{p}$ of $\mathrm{LaAuAl}_{3}$. Assuming that our system behaves as a simple two level model with an energy splitting of $k_{\mathrm{B}} T_{K}$ [27], we can evaluate a $T_{\mathrm{K}}$ of about $3.7 \mathrm{~K}$, close to a previous estimation of $T_{\mathrm{K}}=4.5 \mathrm{~K}$ [21].

Now, we analyze the field dependent of $\gamma_{\text {elec }}$ presented in Fig. 3 based on a theoretical model, which was proposed to explain the field dependent of effective mass $\left(\mathrm{m}^{*}\right)$ of quasiparticles observed from the de Haas-van Alphen effect (dHvA) study for HF systems by Wasserman et al. [28]. Further, Rasul [29] has shown that the mass enhancement occurring in the dHvA amplitude is the same as that found in the heat capacity and the results are in agreement with experiments on $\mathrm{CeB}_{6}$ [28]. Following Wasserman et al. [28], the expression for the field dependent $\gamma_{\mathrm{elec}}\left(\mu \mathrm{m}^{*}\right)$ can be written as follows:

$$
\gamma_{\mathrm{elec}}(H)=\gamma_{0}\left(1+\left(2 \mathrm{D} n_{f} T_{\mathrm{K}}\right) /\left(N\left(T_{\mathrm{K}}+g \mu_{\mathrm{B}} J H\right)^{2}\right)\right)
$$

Here, $\gamma_{0}$ is free-electron linear term of heat capacity, which is proportional to band mass $\left(m_{\mathrm{b}}\right), 2 \mathrm{D}$ is the conduction electron band width, $n_{f}$ is the mean occupancy of $4 f$ electron (for $\mathrm{Ce}^{3+}$ state $\left.n_{f} \sim 1\right), N$ is the effective spin degeneracy of the conduction electrons and local $f$ electrons (the magnetic field lifts only the spin degeneracy of these electrons), $T_{\mathrm{K}}$ is Kondo temperature, $g$ is the electron $g$ factor, $\mu_{\mathrm{B}}$ is the Bohr magneton, $J$ is angular momentum of $f$ electrons (which is related to the angular momentum $m$ of the conduction electrons by $m=-J$, where $H$ is the applied magnetic field). As assumed in the analysis of the field dependent effective mass of $\mathrm{CeB}_{6}$ [28], we have used $N=2, J=5 / 2$, and further we used $g=6 / 7$ for $\mathrm{Ce}^{3+}$ state. Hence, we are left with three variables, $\gamma_{0}, 2 D n_{f}$, and $T_{\mathrm{K}}$. Keeping $2 D n_{f}=0.5 \mathrm{eV}$, we varied $\gamma_{0}$ and $T_{\mathrm{K}}$, and the best fit to the data was obtained for $\gamma_{0}=5.30(2) \times$ $10^{-4}\left(\mathrm{~J} / \mathrm{mole}-\mathrm{K}^{2}\right)$ and $\mathrm{T}_{\mathrm{K}}=4.0(3) \mathrm{K}$ (quality of the fit can be seen in Fig. 3 shown by the solid line). Further, the validity of our analysis is also supported through a very similar value of $T_{\mathrm{K}}$ estimated from our INS study discussed in Sec. III G.

\section{Magnetic susceptibility of $\mathrm{CeAuAl}_{3}$}

Figure 4(a) shows the ac susceptibility for $633 \mathrm{~Hz}$ between $90 \mathrm{mK}$ to $3.5 \mathrm{~K}$ (red color) and ZF cooled dc susceptibility at $10^{-3} \mathrm{~T}$ (black color) between 3.5 to $300 \mathrm{~K}$. The ac susceptibility values are calibrated by using the low temperature values of the dc susceptibility between 1.8 and $3.5 \mathrm{~K}$, as mentioned in Sec. II. Figure 4(a) shows one clear magnetic transition at $T_{\mathrm{N}}=1.1 \mathrm{~K}$ in agreement with published studies [20,21] and the possibility of another transition near $0.18 \mathrm{~K}$, which needs further investigation. Figure 4(a) also shows the temperature dependence of the $T \times \chi$ for which the magnetic transitions temperatures are much better observed. Both transitions did not reveal any systematic shift with frequency and respond within a normal linear regime on increasing the amplitude

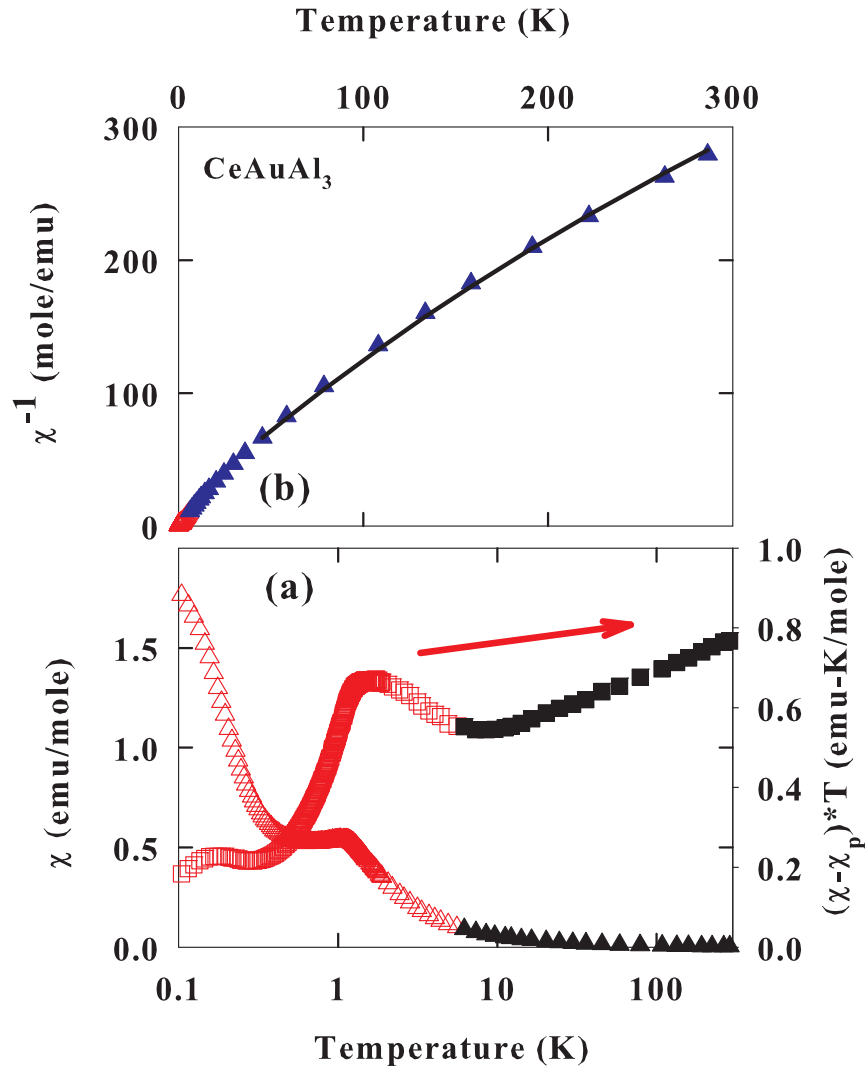

FIG. 4. (Color online) (a) Bottom-left axes: ac (red color open triangles below $3 \mathrm{~K}, v=633 \mathrm{~Hz}, \sim 10^{-3} \mathrm{~T}$ ) and $\mathrm{ZF}$ cooled $\mathrm{dc}$ magnetic susceptibilities (black color solid triangles at $10^{-3} \mathrm{~T}$ ) of $\mathrm{CeAuAl}_{3} . \chi T$ (solid and open squares) is plotted together with $\chi$ by using the same $\log -\mathrm{T}$ scale for a better appreciation of the two magnetic transitions existing in $\mathrm{CeAuAl}_{3}$. (b) The thermal dependence ( $T$-linear) of the reciprocal magnetic susceptibility, $1 / \chi$, is shown (solid triangles). The blue continuous line represents the best fit after analyzing the CEF effects from the INS experimental results (see Sec. III G).

of the oscillating magnetic field. The thermal dependence of the reciprocal susceptibility $1 / \chi$ is represented in Fig. 4(b). It shows a typical Curie-Weiss $(\mathrm{CW})$ law ( $T$-linear scale) with a negative $\mathrm{CW}$ temperature, $\theta_{\mathrm{p}}=-9.8(2) \mathrm{K}$, and an effective magnetic moment, $\mu_{\mathrm{eff}}=2.50(1) \mu_{\mathrm{B}}$, relatively close to $2.53 \mu_{\mathrm{B}}$ of $\mathrm{Ce}^{+3}$. The estimated temperature independent Pauli contribution, $\chi_{\mathrm{P}} \cong 9.0(01) \times 10^{-4} \mathrm{emu} /$ mole. The deviation from a $\mathrm{CW}$ behavior at low temperature (below $50 \mathrm{~K}$ ) reveals the existence of CEF effects, which are well documented in the literature [20,21]. However, here we provide direct confirmation of the $\mathrm{CEF}$ in $\mathrm{CeAuAl}_{3}$ by using the INS measurements that will be presented below.

\section{Magnetization and electrical resistivity}

Figure 5(a) shows the field dependence of high-field magnetization isotherms up to $9 \mathrm{~T}$ between 1.8 and $50 \mathrm{~K}$ for $\mathrm{CeAuAl}_{3}$. The magnetization isotherms show a different field dependence at around $10 \mathrm{~K}$ within the paramagnetic phase. The magnetization isotherms tend to saturate for cooling down to $1.8 \mathrm{~K}$ [see Fig. 5(a), but they are linear above $10 \mathrm{~K}$. The 

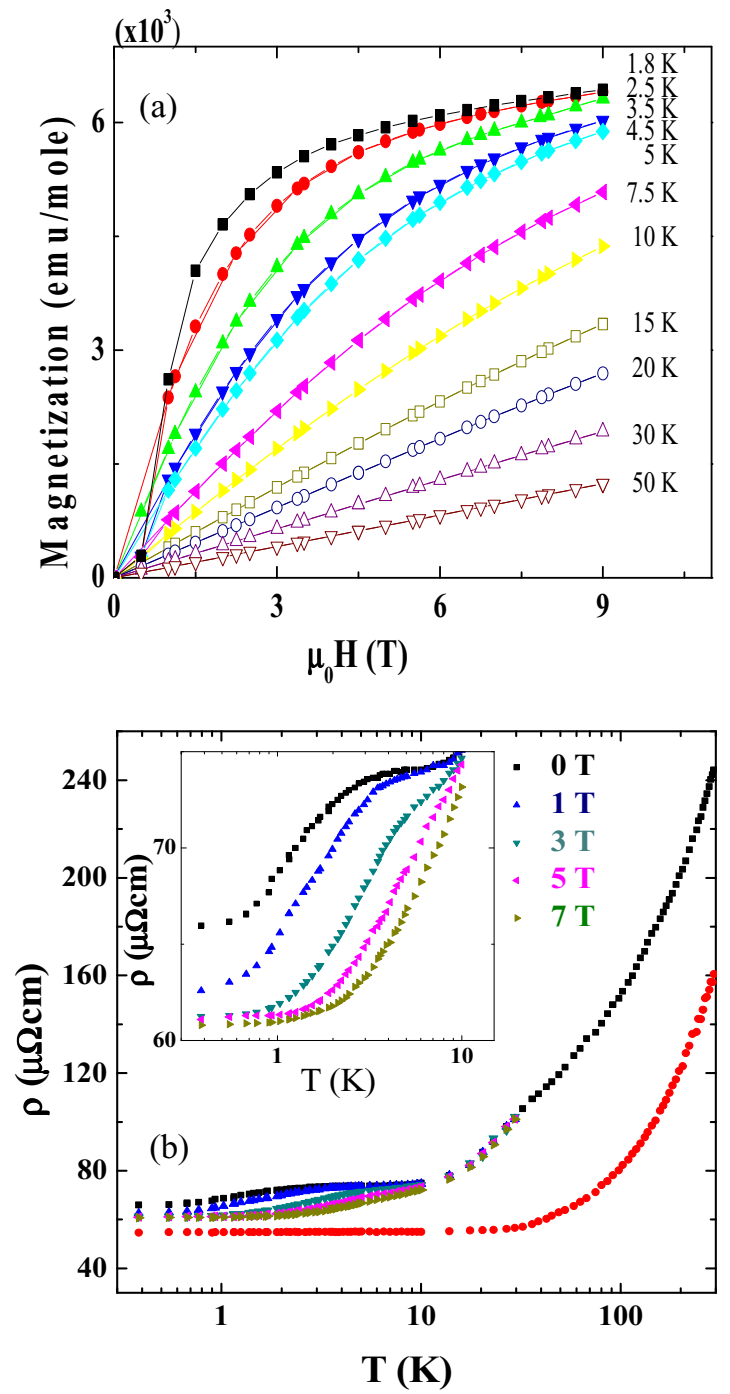

FIG. 5. (Color online) (a) Magnetic field dependence of magnetization between 1.8 and $50 \mathrm{~K}$, and up to $9 \mathrm{~T}$ for $\mathrm{CeAuAl}_{3}$. (b) The thermal dependence of the electrical resistivity measured from 0.35 to $10 \mathrm{~K}$ and up to $7 \mathrm{~T}(\boldsymbol{\square} \mathrm{T}, \boldsymbol{\Delta} 1 \mathrm{~T}, \nabla 3 \mathrm{~T},\langle 5 \mathrm{~T}$, and $>7 \mathrm{~T})$ for $\mathrm{CeAuAl}_{3}$. The $\mathrm{ZF}$ thermal dependence of $\rho$ for $\mathrm{LaAuAl}_{3}$ is shown for comparison (red circles and bottom curve). Inset in (b) shows the data in expanded scale.

saturation type behavior could be due to the CEF effect or a presence of short range magnetic interactions above $T_{\mathrm{N}}$. No magnetic remanence is observed. The magnetic moment at $1.8 \mathrm{~K}, \cong 1.3 \mu_{\mathrm{B}} /$ f.u., can be calculated from magnetization at 9 T. Figure 5(a) shows a metamagnetic-type transition around $0.5 \mathrm{~T}$ at $1.8 \mathrm{~K}$, close to $T_{\mathrm{N}}$. The overall low temperature behavior of the magnetization can be explained based on CEF effects along with magnetic exchange.

Figure 5(b) displays the thermal dependence from 0.35 to $300 \mathrm{~K}$ of the electrical resistivity for $\mathrm{CeAuAl}_{3}$ up to a $7 \mathrm{~T}$ applied field and for $\mathrm{LaAuAl}_{3}$ at $0 \mathrm{~T}$. The resistivity for $\mathrm{CeAuAl}_{3}$ shows a linear decrease from 300 to $\approx 100 \mathrm{~K}$ and a small plateau between 8 and $4 \mathrm{~K}$. Anomalies at around $10-50 \mathrm{~K}$ are considered as coming from the influence of CEF. Both compounds show an average ratio, $\rho(300 \mathrm{~K}) /$ $\rho(0.35 \mathrm{~K}) \approx 3.8$, which could indicate a slight structural

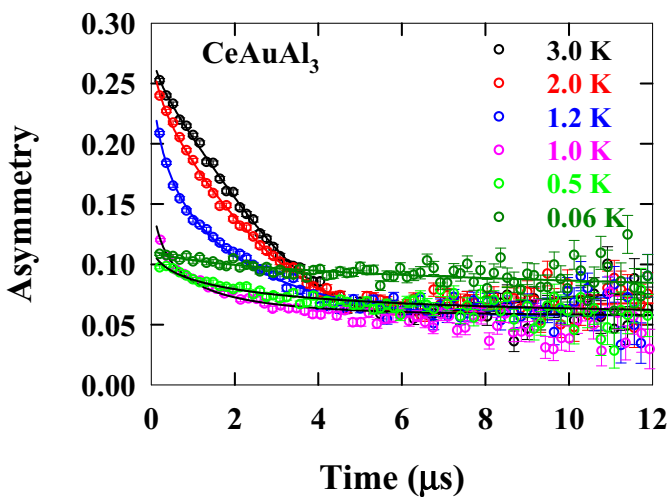

FIG. 6. (Color online) ZF $\mu$ SR spectra plotted as asymmetry versus time at various temperatures between 0.55 and $3 \mathrm{~K}$ from $\mathrm{CeAuAl}_{3}$. The lines are least squares fits (see text) to the data using Eq. (2).

disorder, as the structural analysis has shown in Sec. II (see Table I). The inset of Fig. 5(b) shows the low temperature region on an expanded scale; however, there is no sharp transition seen near $T_{\mathrm{N}}$, but a small change in the slope has been observed that is in agreement with the published results [20]. As shown in Fig. 2 for $C_{p}$, the transition in the field dependent $\rho$ is slightly shifted to higher temperatures with the applied magnetic field. The $\mathrm{LaAuAl}_{3}$ resistivity was used to calculate: (1) the phonon contribution from other impurity contributions $(\approx 54.8 \mu \Omega \mathrm{cm}$ ), a Debye temperature of $\approx 170 \mathrm{~K}$ (by using a Bloch-Grüneisen-Mott law) [20], and (2) the magnetic contribution $\Delta \rho$ to the electrical resistivity $\rho$. Figure 3 (right $y$ axis) shows the coefficient $A$ of $T^{2}$ contribution $\left(\Delta \rho \sim A T^{2}\right)$ of the magnetic resistivity as a function of applied magnetic field. It shows that the field dependence of $A$ is very close to the field dependence of $\gamma_{\text {elec }}$ and hence a similar theoretical model can be applied to understand the field dependent of $A$ as applied for $\gamma_{\text {elec }}$ in Sec. III B.

\section{E. Muon spin relaxation}

To shed light on the two-phase transitions seen in the ac susceptibility, we have investigated the temperature dependence of the $\mu \mathrm{SR}$ in ZF. Figure 6 shows the ZF asymmetry $\mu \mathrm{SR}$ spectra of $\mathrm{CeAuAl}_{3}$ at selected temperatures between 0.05 and $3 \mathrm{~K}$. At $3 \mathrm{~K}$, the $\mu \mathrm{SR}$ spectra exhibit a typical behavior expected from the static nuclear moment. The ZF $\mu$ SR spectra were fitted using a static Gaussian Kubo-Toyabe (GKT) function [30] multiplied by an exponential decay under a constant ground $A_{\text {gnd }}$,

$$
\begin{aligned}
G_{z}(t)= & A_{0}\left(\frac{1}{3}+\frac{2}{3}\left(1-(\sigma t)^{2}\right) \exp \left(-\frac{(\sigma t)^{2}}{2}\right)\right) \\
& \times \exp \left(-(\lambda t)^{\beta}\right)+A_{\mathrm{gnd}},
\end{aligned}
$$

where $A_{0}$ is the initial ZF asymmetry parameter, $\sigma$ is the nuclear contribution, and $\lambda$ is the electronic relaxation rate mainly arising from the local $4 f$ moment of the $\mathrm{Ce}$ ion. The static GKT function results from a Gaussian distribution of local magnetic fields at the muon site that arises from the nuclear spins [30]. The exponential decay, $\exp \left(-(\lambda t)^{\beta}\right)$, is the magnetic contribution that results from the dynamic magnetic 


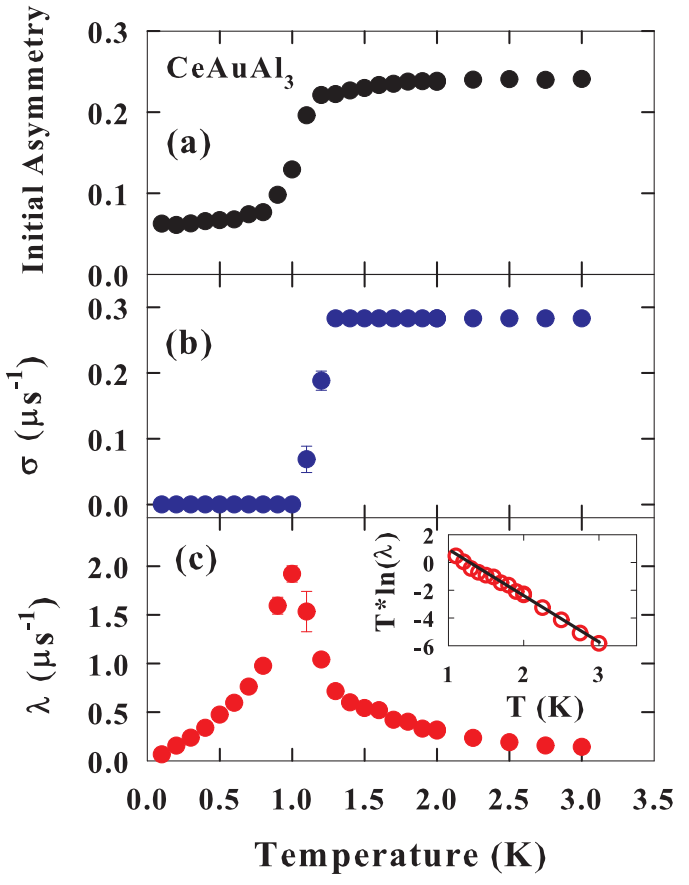

FIG. 7. (Color online) Temperature dependence of (a) initial asymmetry, $A_{0}$, (b) nuclear contribution, $\sigma$ and (c) electronic relaxation rate, $\lambda$, estimated from the fit using Eq. (2). Inset in (c) shows $T \ln (\lambda)$ vs $T$, with the solid being a fit (see text) to Eq. (3).

fields, which arise from the fluctuating electronic spins. The multiplicative nature of the nuclear and magnetic contributions is only valid if these processes are independent, as it was assumed in our case. We had estimated the value of $A_{\text {gnd }}$ and $\beta \sim 0.5$ from the fit of $3 \mathrm{~K}$ data, and these values were kept fixed to reduce the number of fit parameters. Further, the value of $\sigma$ was also estimated from $3 \mathrm{~K}$ data, was kept fixed between 3 and $1.2 \mathrm{~K}$, and was allowed to vary below $1.2 \mathrm{~K}$. Figures 7(a)-7(c) show the temperature dependence of $A_{0}, \sigma$, $\lambda$ parameters obtained after fitting the ZF- $\mu$ SR spectra. It is clear that at $1.1 \mathrm{~K}, A_{0}$, and $\sigma$ exhibit a sharp drop, while $\lambda$ exhibits a peak. $A_{0}$ drops nearly to $\frac{1}{2}$ of its high temperature values [Fig. 7(a)] indicating the bulk nature of the long-range magnetic ordering as seen through the heat capacity and ND (discussed in Sec. III F). In the ordered state, the internal fields arising from the electronic moment ordering are high compared to those from the nuclear moment. Hence, the muons mainly sense the $4 f$-electronic magnetic field below $T_{\mathrm{N}}$, and as a result, $\sigma$ cannot be measured (or nuclear contribution disappears compared with electronic contribution), which is seen in Fig. 7(b). The absence of any frequency oscillations in the $\mu \mathrm{SR}$ spectra at $0.055 \mathrm{~K}$ (i.e., below $T_{\mathrm{N}}$ ) indicates that internal fields at the muon sites are high and outside the time windows of the $\mu$ SR spectrometer. This limitation is due to the broad pulse width $(\sim 80 \mathrm{~ns})$ of the muon pulses at ISIS. Further, the divergence of $\lambda$ [see Fig. 7(c)] above $T_{\mathrm{N}}$ also confirms that the transition is magnetic and magnetic moment fluctuations start slowing down well above $T_{\mathrm{N}}$. Note that the transition temperature estimated by $\mu \mathrm{SR}$ is in agreement with that estimated by $C_{p}$ and susceptibility measurement, but the drop in the asymmetry occurs over a relative wide temperature range, $\Delta T=0.36 \mathrm{~K}$, may suggest a distribution of the ordering temperature or broad nature of the transition. It is to be noted that $\lambda(T)$ relative to $T_{\mathrm{N}}$ exhibits symmetric behavior, which might be related to the slow down of the critical magnetic fluctuations. It is also of interest to note that the temperature dependence of $\lambda$ shows a clear Arrhenius-like behavior [see the inset of Fig. 7(c)], i.e.,

$$
\lambda=\lambda_{0} \exp \left(\frac{-E_{a}}{k_{\mathrm{B}} T}\right)
$$

where $E_{a}$ is an activation energy and $k_{\mathrm{B}}$ is the Boltzmann constant. This shows that the spin dynamics within $\mathrm{CeAuAl}_{3}$ are based on a thermally activated process with a barrier energy of $E_{a}=0.004(1) \mathrm{K}$ and $\lambda_{0}=0.077(8) \mu s^{-1}$. This shows that the spin dynamics of $\mathrm{CeAuAl}_{3}$ is a thermally activated process with a very small energy barrier. This type of activation behavior has been observed for $\mathrm{CeInPt}_{4}$ with $E_{a}=0.0029 \mathrm{~K}$, which remains paramagnetic down to $0.040 \mathrm{~K}$ [31]. In order to decouple the nuclear contribution from the electronic contribution, we also measured the temperature dependent $\mu$ SR spectra in an applied longitudinal field of $5 \times 10^{-3} \mathrm{~T}$. The data were fitted with Eq. (2) but without the KT term (i.e., $\sigma=0$ ). The temperature dependent $A_{0}$ and $\lambda$ (not shown here) are also in agreement with those values given in Fig. 7. In order to obtain an estimate of the internal field at the muon sites, we also measured the field dependence of $\mu \mathrm{SR}$ spectra for applied fields between 0 and $0.25 \mathrm{~T}$ at $0.06 \mathrm{~K}$. The initial asymmetry increases with the field and reaches 0.20 at a field of $0.25 \mathrm{~T}$ compared to a value of 0.26 at $3 \mathrm{~K}$ in $\mathrm{ZF}$, which indicates that the internal fields on the muon sites are larger than $0.25 \mathrm{~T}$. As it was not possible to get information about the magnetic structure of $\mathrm{CeAuAl}_{3}$ from our $\mu \mathrm{SR}$ study, we therefore carried out a ND study, and the results are presented in the next section.

\section{F. Magnetic structure using ND}

Figure 8 shows the ND data collected at $0.3 \mathrm{~K}$ for the 10 degree (bank 1) and 35 degree (bank 3) detector banks on GEM. At $0.3 \mathrm{~K}$, extra Bragg peaks are observed compared with $2 \mathrm{~K}$ data (not shown here). Their intensities as a function of $Q$ (stronger at smaller $Q$ and falling towards higher $Q$ ) indicate that these are due to the long-range magnetic ordering of the Ce-moment. For the estimation of the magnetic propagation vector, an automatic indexing procedure using a grid search in the FullProf program was used [32]. The ND data allow for a direct observation of the propagation vector compared to indirect measurement of the propagation vector by NMR [22]. In principle, the propagation vector can be refined in the Rietveld fitting process, but uncertainties of the zero-shift of GEM detector bank 1 at a $d$-spacing of 20 and the small number of weak magnetic reflections in other detector banks hampered variation in our case. Therefore, the propagation vector was manually adjusted until the observed extra peaks were successfully indexed using $\mathbf{k}=(0,0,0.52(1))$, which is close to $(0,0,0.55)$ proposed by the NMR study [22].

A symmetry analysis using the program SARAh [33] for an incommensurate structure with $\mathbf{k}=(0,0,0.52)$ for $\mathrm{Ce}$ atoms at $(0,0,0)$ indicates that there are four, one-dimensional (1D) 

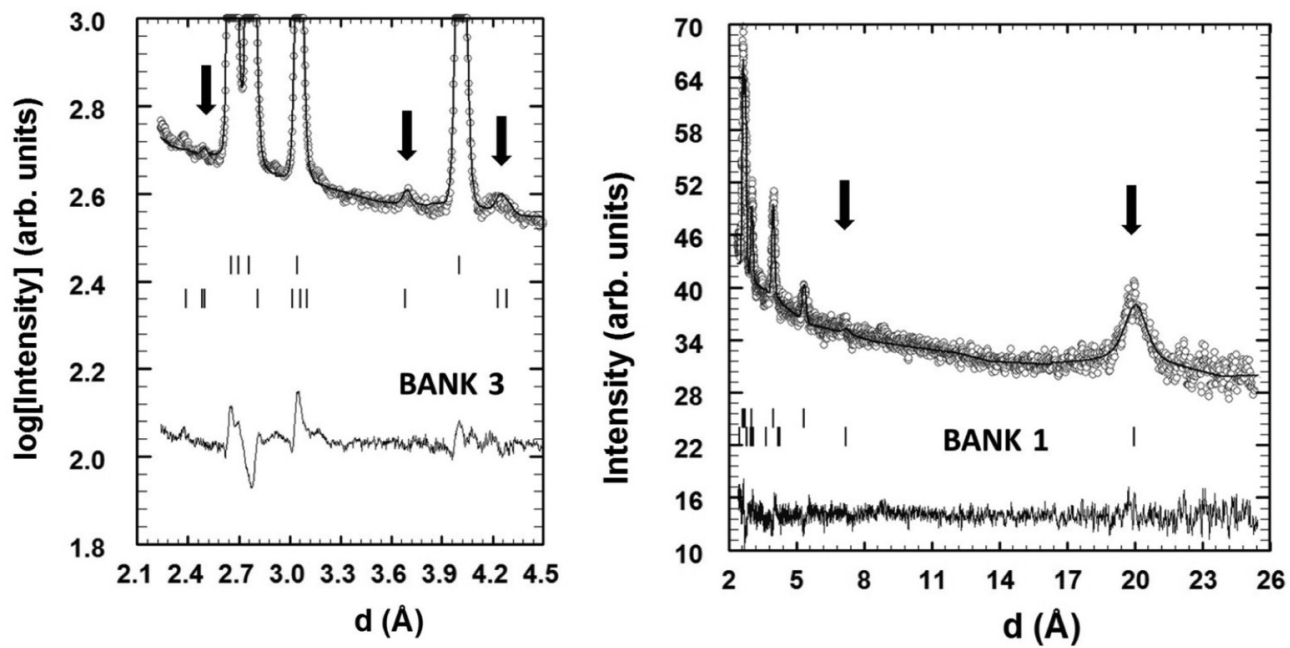

FIG. 8. Rietveld fitted ND patterns collected at $0.3 \mathrm{~K}$ for $\mathrm{CeAuAl}_{3}$. The magnetic peaks are marked with arrows. The circle symbols show the experimental data, and the solid line shows the fit and the line plot at bottom is the difference between them. The vertical tick marks indicate the positions of Bragg peaks for the nuclear scattering (top) and for the magnetic scattering (bottom) with propagation vector $\mathbf{k}=(0,0,0.52(1))$

representations, labeled $\Gamma_{1}$ to $\Gamma_{4}$, and one two-dimensional (2D) representation $\Gamma_{5}$ in the little group. Only $\Gamma_{2}$ and $\Gamma_{5}$ enter the decomposition of $\Gamma_{\text {mag }}=\Gamma_{2}+\Gamma_{5} . \Gamma_{2}$ and $\Gamma_{5}$ correspond, respectively, to an ordering of the $\mathrm{Ce}$ site along the $c$ axis (one component, imposing a sinusoidal structure) and in the $a b$ plane (two basis vectors with real and imaginary components along $a$ and $b$, each of both enabling spiral arrangements rotating in opposite directions, or if linked together, an helicoidal structure with an elliptical envelop controlled by the linear combination of the two vectors). A good fit to the data (magnetic Bragg factor for bank $1 R_{\mathrm{B}}=6 \%$ ) was obtained using FullProf [32] with a single basis vector of the representation $\Gamma_{5}$ (see Fig. 8, solid line). The fit using $\Gamma_{2}$ was not able to explain the intensities of the observed magnetic peaks, as expected from the NMR results [22]; in particular, $\Gamma_{2}$ does not contribute to the strongest magnetic peak at $20 \AA$. The magnetic structure of $\mathrm{CeAuAl}_{3}$ is hence a simple helicoidal structure (Fig. 9), for which Ce moments are ferromagnetically aligned in the $a b$ plane and for which moments rotate by an angle in radians given by $\varphi=2 \pi \times K \times t$, where $t$ is a translation along the $c$ direction. For magnetic moments in neighboring planes containing $\mathrm{Ce}$-atoms at $(0,0,0)$ and at the centering translation $\left(\frac{1}{2}, \frac{1}{2}, \frac{1}{2}\right)$, respectively, the rotation angle is $\varphi=93.6^{\circ}$, in agreement with the model proposed using NMR results. The magnetic moment is $1.05(09) \mu_{\mathrm{B}}$ in the $a b$ plane. The relatively large error of the Ce-moment is due to the magnetic structure analysis being dominated by the strong magnetic Bragg reflection at $20 \AA$, a $d$-spacing region, which on the GEM diffractometer is affected by a systematic error of typically $10 \%$ due to low neutron count rates and uncertainties of the wavelength-dependent neutron flux determination. The direction and absolute value of the magnetic moment is compared to the estimated moment from the CEF analysis in the next section. Our attempt to fit data with a commensurate propagation vector $\mathbf{k}=\left(\begin{array}{lll}0 & 0 & \frac{1}{2}\end{array}\right)$ did not give a good fit, which is in agreement with NMR study.
It is worth comparing the magnetic structure and the direction of the magnetic moments of $\mathrm{CeAuAl}_{3}$ to those of isostructural compounds, $\mathrm{CeCuAl}_{3}$ and $\mathrm{CeAgAl}_{3}$. The compound $\mathrm{CeCuAl}_{3}$ exhibits an $\mathrm{AFM}$ ordering at $T_{\mathrm{N}}=2.5 \mathrm{~K}$ with a propagation vector $\mathbf{k}=(0.5,0.5,0)$ and moment along the $c$ axis [14], while $\mathrm{CeAgAl}_{3}$ is a FM with $T_{C}=3 \mathrm{~K}$ [15] and easy magnetization axis in the $a b$ plane. Note that even though the $\mathrm{Cu}, \mathrm{Ag}$, and $\mathrm{Au}$ are isoelectronic, the magnetic properties of these compounds are dramatically different, which might indicate that magnetic exchanges, controlled through magnetostriction, magnetovolume pressure, and chemical pressure, play an important role in determining the ground states of these compounds.

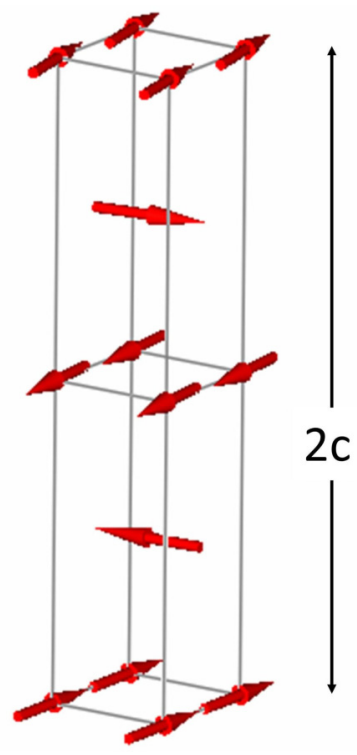

FIG. 9. (Color online) Magnetic structure of $\mathrm{CeAuAl}_{3}$ at $0.3 \mathrm{~K}$ along two unit cells along the $c$ direction. 


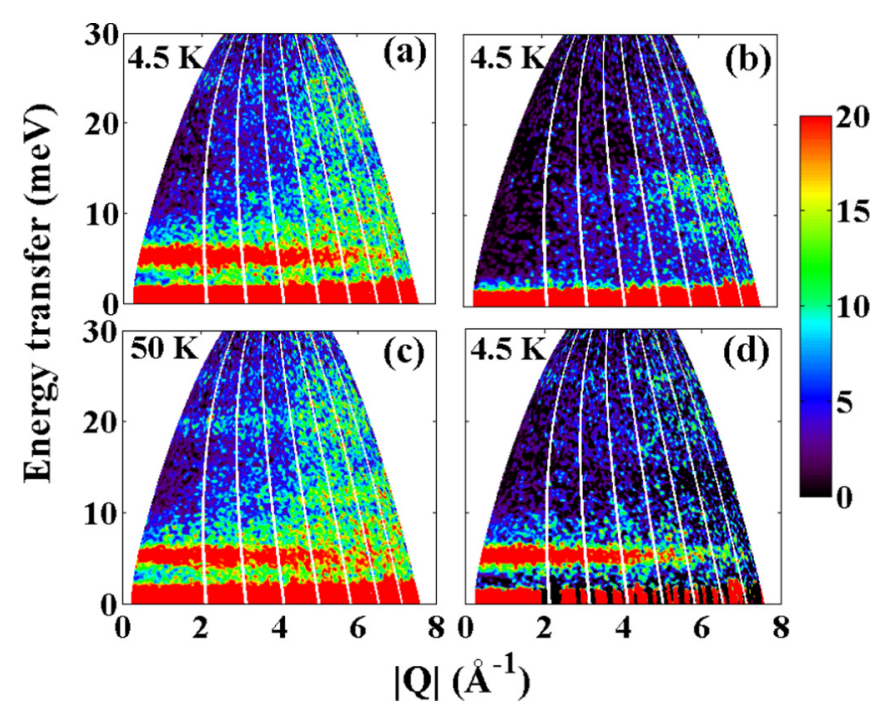

FIG. 10. (Color online) 2D contour plots of the spectral function $S(Q, \omega)$ (a) of $\mathrm{CeAuAl}_{3}$ at $4.5 \mathrm{~K}$ and (c) at $50 \mathrm{~K}$, (b) of $\mathrm{LaAuAl}_{3}$ at $4.5 \mathrm{~K}$ and (d) estimated magnetic scattering of $\mathrm{CeAuAl}_{3}$ at $4.5 \mathrm{~K}$ after subtracting phonon scattering using $\mathrm{LaAuAl}_{3}$ data.

\section{G. Inelastic neutron scattering}

Figures 10(a)-10(d) show the inelastic neutron spectral function $S(Q, \omega)$ as $2 \mathrm{D}$ contour plots and energy transfer versus wave vector transfer, for $\mathrm{CeAuAl}_{3}$ [Fig. 10(a) for $4.5 \mathrm{~K}$ and Fig. 10 (c) for $50 \mathrm{~K}$ ] and $\mathrm{LaAuAl}_{3}$ [Fig. 10(b) for $4.5 \mathrm{~K}$ ] measured with the incident neutron energy $E_{i}=35 \mathrm{meV}$. Figure $10(d)$ shows the magnetic scattering from $\mathrm{CeAuAl}_{3}$ at $4.5 \mathrm{~K}$ after subtracting the phonon contribution obtained from the $\mathrm{LaAuAl}_{3}$ data. It is clear from Figs. 10(a)-10(d) that $\mathrm{CeAuAl}_{3}$ exhibits two magnetic excitations near 5.1 (very strong) and 24.7 (weak) meV at low $Q$, while there is only weak phonon scattering in $\mathrm{LaAuAl}_{3}$ at these energies at low $Q$. The excitations arise from the splitting of the $J=5 / 2$ ground multiplet under the crystal field potential, which gives three doublets in the paramagnetic state. The low energy excitation at $5.1 \mathrm{meV}$ is observed very clearly at $4.5 \mathrm{~K}$; however, the other at $\approx 24.7 \mathrm{meV}$ is very weak due to the small matrix elements between the ground state and the highest lying doublet. Furthermore, when the temperature was raised to $50 \mathrm{~K}$, a clear new excitation appears near $20 \mathrm{meV}$ [see Fig. 10(c)], which is the excited state transition from the first CEF doublet near $5.1 \mathrm{meV}$ to this highest CEF doublet near $24.7 \mathrm{meV}$. The high $Q$ phonon contributions of $\mathrm{CeAuAl}_{3}$ and $\mathrm{LaAuAl}_{3}$ at 4.5 and $250 \mathrm{~K}$ are quite similar, which can be seen in the $1 \mathrm{D}$ cuts made from the $2 \mathrm{D}$ color plots, as plotted in Figs. 11(a)-11(f).

In Figs. 11(a)-11(f), we have also plotted $Q$-integrated energy cuts at low $Q\left(0\right.$ to $\left.3 \AA^{-1}\right)$ and at high $Q\left(5\right.$ to $\left.8 \AA^{-1}\right)$ for $\mathrm{CeCuAl}_{3}$ and $\mathrm{LaCuAl}_{3}$ at several temperatures, which again confirm that there is a very small phonon contribution compared to the magnetic signal, especially near $5 \mathrm{meV}$ and at low $Q$. Thus, we have directly subtracted the data of $\mathrm{LaAuAl}_{3}$ from that of $\mathrm{CeAuAl}_{3}$ to estimate the magnetic scattering, $S_{\mathrm{M}}(Q, \omega)$, in $\mathrm{CeAuAl}_{3}$. The intensities of the excitations near $5 \mathrm{meV}$ decrease on increasing $Q$ following the square of the magnetic form factor $F(Q)$ for a $\mathrm{Ce}^{3+}$ ion (figure not shown

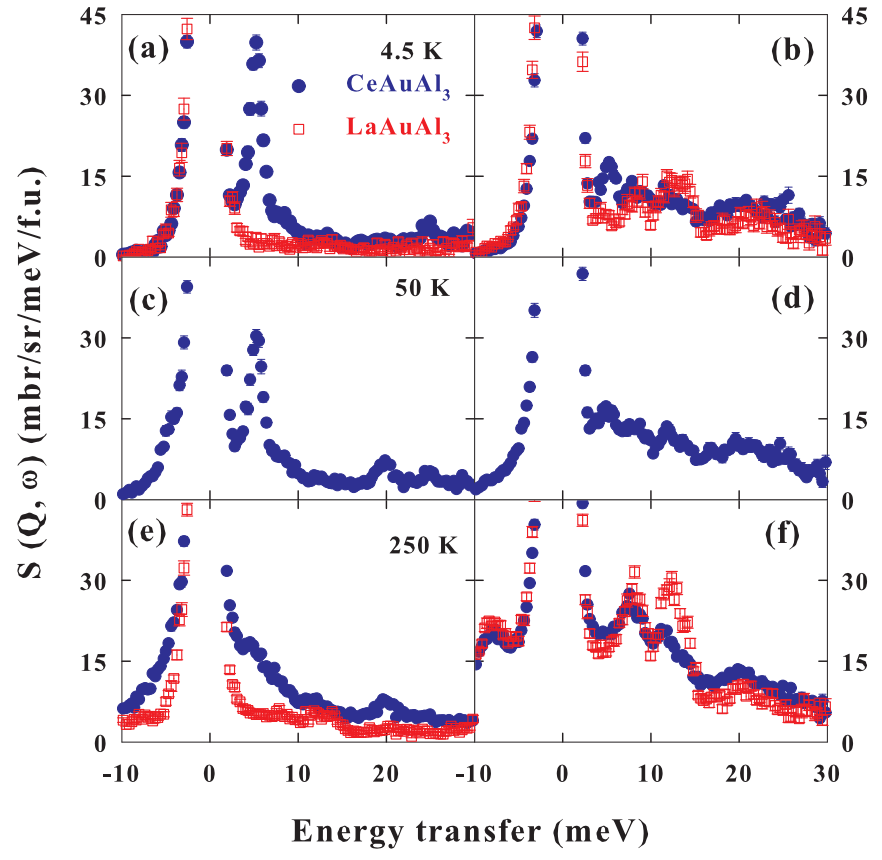

FIG. 11. (Color online) The $Q$-integrated 1D cuts of the total scattering from $\mathrm{CeAuAl}_{3}$ (blue circles) and $\mathrm{LaAuAl}_{3}$ (open red squares) at low $Q\left(Q=2.43 \AA^{-1}\right)$ at $4.5 \mathrm{~K}$ (a), $50 \mathrm{~K}$ (c), and $250 \mathrm{~K}$ (e). (b), (d), and (f) show the $1 \mathrm{D}$ cuts at high $Q\left(Q=6.43 \AA^{-1}\right)$ revealing mainly the phonon contribution.

here). The observed small deviation from the $F^{2}(Q)$ behavior could arise due to imperfect subtraction of phonon contribution, background coming from the closed-cycle refrigerator (CCR), and/or presence of short range magnetic correlations above the magnetic ordering temperature. Further, the intensity of the $24.7 \mathrm{meV}$ peak also decreases on increasing $Q$ up to $4 \AA^{-1}$ and then remains nearly constant. As the measured intensity of this peak is very small and due to the presence of phonon scattering at the same position, it was not possible to give any qualitative $Q$-dependent analysis for this excitation by using the $\mathrm{Ce}^{3+}$ form factor $F^{2}(Q)$.

Now, we present the analysis of the estimated magnetic scattering at 4.5, 50, and $250 \mathrm{~K}$ [see Figs. 12(a)-12(c)], based on the CEF theory and Kramer's theorem for the $\mathrm{Ce}^{3+}$ ion $\left(4 f^{1}\right)$. In this way, we will achieve a full characterization of the CEF effects on the heat capacity and magnetic susceptibility indicated at the end of this section.

Two magnetic excitations at around 5.1 and $24.7 \mathrm{meV}$ have half-linewidths of 0.71 (4) $\mathrm{meV}$ and 1.18 (11) $\mathrm{meV}$, respectively, at $4.5 \mathrm{~K}$ suggesting that our sample is in a well crystallographically ordered state, which is in agreement with our diffraction analysis discussed above. Further, the smaller linewidth of the CEF excitations suggests that the hybridization between localized $4 f^{1}$ electronic states and conduction electrons must be smaller, which is in agreement with the reported smaller value of the Kondo temperature, $T_{\mathrm{K}}=4.5 \mathrm{~K}[20,21]$. We will develop this point further using low energy INS data.

The CEF Hamiltonian for a tetragonal point symmetry $\left(C_{4 v}\right)$ of the $\mathrm{Ce}$ ion in $\mathrm{CeAuAl}_{3}$ can be written as $H_{\mathrm{CEF}}=$ $B_{2}^{0} O_{2}^{0}+B_{4}^{0} O_{4}^{0}+B_{4}^{4} O_{4}^{4}$, where $B_{n}^{m}$ and $O_{n}^{m}$ are the CEF 


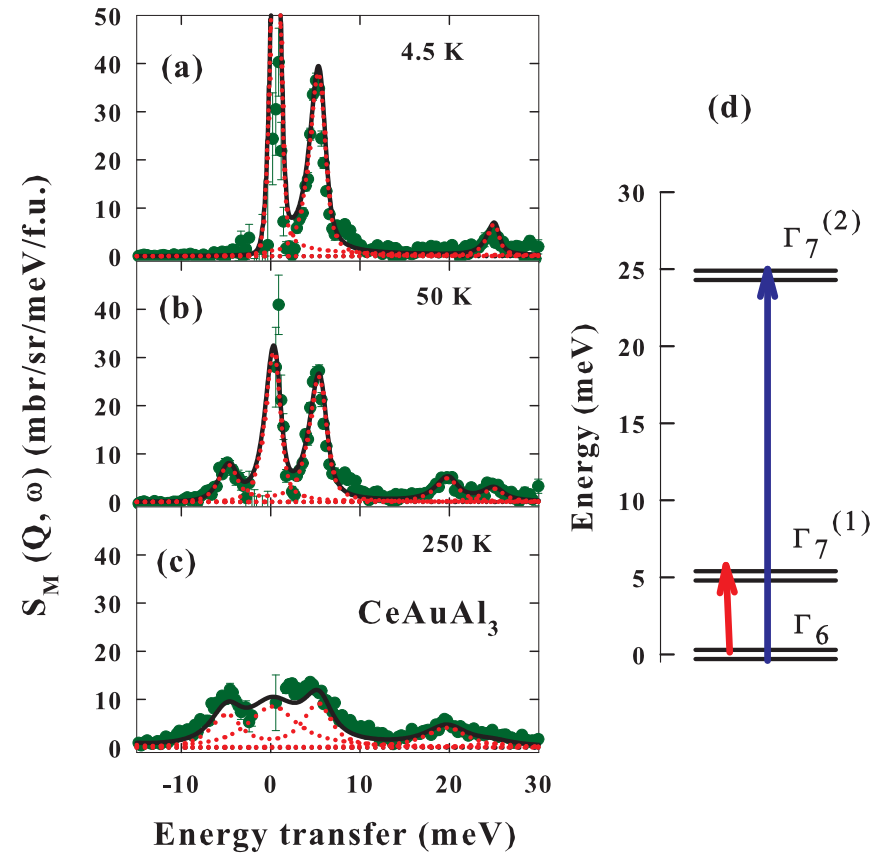

FIG. 12. (Color online) The estimated magnetic scattering from $\mathrm{CeAuAl}_{3}$ at low $Q=2.43 \AA^{-1}$ at (a) $4.5 \mathrm{~K}$, (b) $50 \mathrm{~K}$, and (c) $250 \mathrm{~K}$. The thick solid lines represent the fits (based on the CEF model), and dotted lines show the components of the fits (see text). (d) The schematic CEF level scheme (three CEF doubles at 0, 5.1, and $24.6 \mathrm{meV}$ ) of the $\mathrm{Ce}^{3+}$ ions in $\mathrm{CeAuAl}_{3}$ deduced from the INS experiment (see text). The transitions from the ground state to the excited states that contribute to the observed excitations are shown by arrows.

parameters and Steven's operators, respectively [34,35]. The sixfold degenerate $\mathrm{Ce}^{3+}(J=5 / 2)$ states, $4 f^{1}$, split into three doublets (Kramer's theorem establishes that for odd numbers of localized electron the minimum degeneracy should be a doublet) in the paramagnetic phase. The CEF parameters were obtained from a simultaneous fit to INS data at 4.5, 50, and $250 \mathrm{~K}$. Considering three CEF parameters to be fitted with two energies and six intensities (two at each temperature), we have obtained a unique set of the CEF parameters. Figure 12 shows the best fit (red solid line) to $4.5,50$, and $250 \mathrm{~K}$ data with the CEF parameters $B_{2}^{0}=1.2208(130) \mathrm{meV}, B_{4}^{0}=$ $-0.0021(3) \mathrm{meV}$, and $B_{4}^{4}=0.2555(2) \mathrm{meV}$. This set of CEF parameters yields eigenvalues of $0,5.1$, and $24.6 \mathrm{meV}$, and the eigenvectors (in Bethe's notation) are $\left|\Gamma_{6} v=\right| \pm 1 / 2$ as ground state, $\left|\Gamma_{7}^{(1)} v=\alpha\right| \pm 5 / 2-\beta \mid \mp 3 / 2$ as first excitation and, $\left|\Gamma_{7}^{(2)} v=\beta\right| \pm 5 / 2+\alpha \mid \mp 3 / 2$ as the second excitation, being $\alpha=-0.375$ and $\beta=0.927$, respectively. The value of $B_{2}^{0}$ can be also determined using the high temperature expansion of the single crystal magnetic susceptibility [36], assuming isotropic exchange. Then, $B_{2}^{0}$ can be written in terms of the CW temperatures, $\theta_{a b}$, when the applied magnetic field is in the $a b$ plane, and $\theta_{c}$ when it is along the $c$ axis. For $\mathrm{CeAuAl}_{3}$, these values are $\theta_{a b}=4.58 \mathrm{~K}$ and $\theta_{c}=-194 \mathrm{~K}$, and they were obtained from the single crystal susceptibility [37] which gives $B_{2}^{0}=20.69 \mathrm{~K}$ (or $1.78 \mathrm{meV}$ ). This value of $B_{2}^{0}$ is larger than that obtained from the INS data, which may indicate the presence of anisotropic exchange interactions in $\mathrm{CeAuAl}_{3}$.

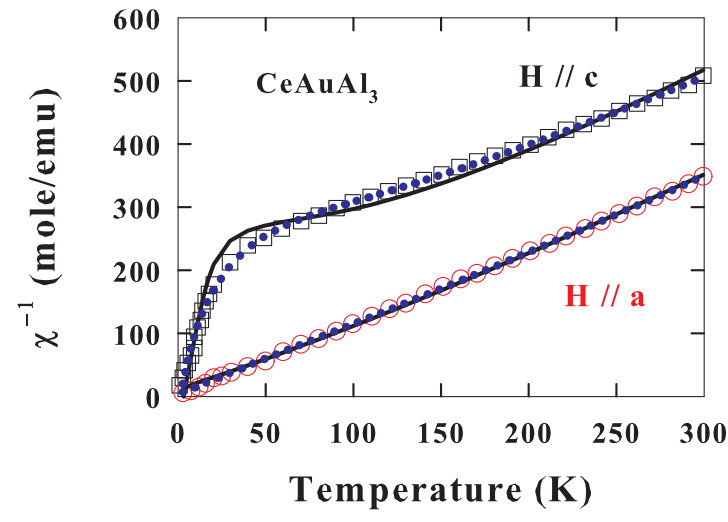

FIG. 13. (Color online) Temperature dependence of the inverse magnetic susceptibility of $\mathrm{CeAuAl}_{3}$ single crystal [37]. The solid black line shows the best fit based on the CEF model including a molecular field parameter with fixed CEF parameters from the INS analysis; the blue dotted line shows the fit with both CEF parameters and molecular field parameters as variables. The latter fit agrees better with the susceptibility data but does not explain the INS data.

The single crystal susceptibility of $\mathrm{CeAuAl}_{3}$ [37] was analyzed using the CEF parameters obtained from the INS analysis enhanced by a molecular field parameter that could describe the intensity of the anisotropy exchange coupling mentioned above. The form of the enhanced susceptibility is given by

$$
\chi^{\xi}=\frac{\chi_{\mathrm{CEF}}^{\xi}}{1+\lambda^{\xi} \chi_{\mathrm{CEF}}^{\xi}}+\chi_{0}^{\xi},
$$

where $\xi=\{\| a$ axis, $\| c$ axis $\}$ and indicate the direction of the applied magnetic field when susceptibility is calculated, $\chi^{\xi} \mathrm{CEF}$ is the single ion susceptibility calculated by using $H_{\mathrm{CEF}}, \lambda^{\xi}$ is the molecular field parameter, and $\chi_{0}^{\xi}$ is a constant temperature independent contribution. Figure 13 shows two fits with Eq. (4). Continuous black lines give the first fit as it is obtained from the CEF parameters obtained previously from the INS analysis. The fit is acceptable for $\chi^{\| a \text { axis }}$ but not adequate for $\chi^{\| c \text { axis }}$ below $50 \mathrm{~K}$. The parameters obtained in this fit are given by $\lambda^{\| a \text { axis }}=$ -7.89(10) (mole $/ \mathrm{emu}), \quad \lambda^{\| c \text { axis }}=46.64(52)(\mathrm{mole} / \mathrm{emu})$, $\chi_{0}^{\| a \text { axis }}=-0.89(2) 10^{-4}(\mathrm{emu} /$ mole $), \quad$ and $\quad \chi_{0}^{\| c \text { axis }}=$ $-1.87(1) 10^{-4}$ (emu/mole). The fit can improve the calculated $\chi^{\| c \text { axis }}$ values at around $50 \mathrm{~K}$ (see, blue dotted points in Fig. 13), as long as CEF parameters can change slightly during the fitting process. In this case, $B_{2}^{0}=1.2036(120) \mathrm{meV}$, $B_{4}^{0}=-0.0031(3) \mathrm{meV}, \quad B_{4}^{4}=0.4269(2) \mathrm{meV}, \quad \lambda^{\| a \text { axis }}=$ $-3.83(8)(\mathrm{mole} / \mathrm{emu}), \quad \lambda^{\| c \text { axis }}=20.25(36)(\mathrm{mole} / \mathrm{emu})$, $\chi_{0}^{\| a \text { axis }}=-0.56(1) \times 10^{-6}(\mathrm{emu} / \mathrm{mole}), \quad$ and $\quad \chi_{0}^{\| c \text { axis }}=$ $-8.490(2) \times 10^{-6}(\mathrm{emu} / \mathrm{mole})$. However, the new set of CEF parameters does not explain the INS results. Although, the CEF parameters estimated from INS data provide a good description of the single crystal susceptibility $\chi^{\| c \text { axis }}$, they point to the existence of a molecular field parameter ruled by an anisotropic indirect exchange, where the strength of the exchange along the $c$ and $a$ axes are quite different and of opposite sign (AFM along the $c$ axis and FM in the $a b$ plane). This is in good agreement with the existence of an anisotropy exchange coupling that stabilizes a helix structure with a stable AFM 


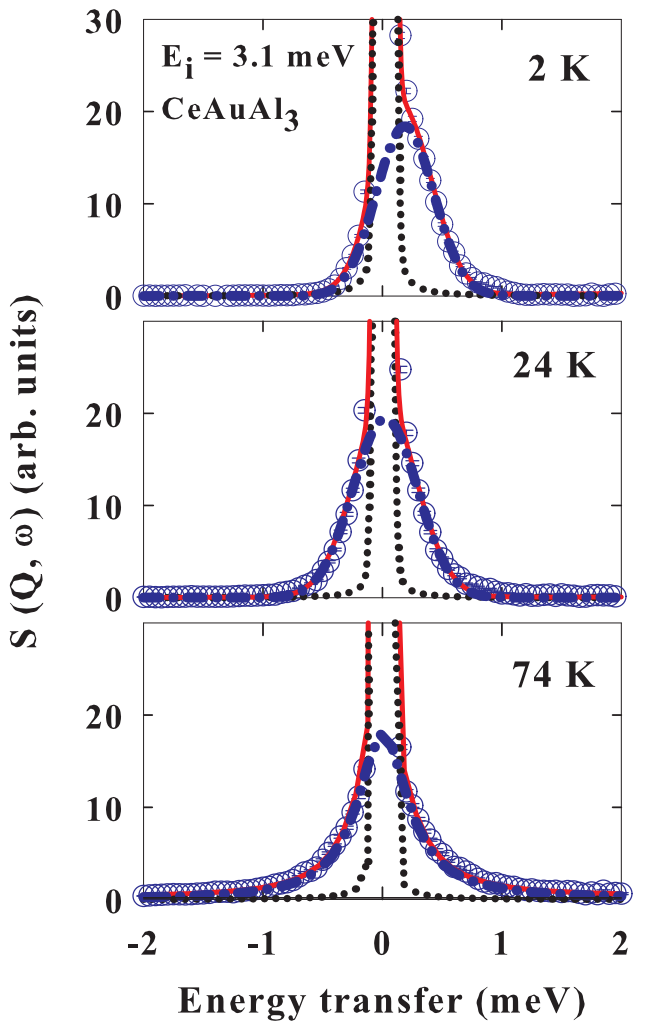

FIG. 14. (Color online) Low energy inelastic response of $\mathrm{CeAuAl}_{3}$ measured at various temperatures with $E_{i}=3.1 \mathrm{meV}$ on IN6. The solid line shows the fit to the data, and the dotted line and dotted-dashed line show the components of the fit (see text for details).

component along the $c$ axis [36]. At this level, using the CEF ground state wave functions, we can calculate the components of magnetic moment for Ce ion in $\mathrm{CeAuAl}_{3},\left\langle\mu_{x}\right\rangle=1.28 \mu_{\mathrm{B}}$ and $\left\langle\mu_{\mathrm{z}}\right\rangle=0.43 \mu_{\mathrm{B}}$ using the CEF ground state wave functions. The large value of $\left\langle\mu_{\mathrm{x}}\right\rangle$ is in agreement with the moment direction (in the $a b$ plane) obtained from ND. Further support of the validity of our CEF analysis comes from the CEF parameters and ground state wave functions estimated using polarization-dependent soft x-ray absorption spectroscopy of $\mathrm{CeAuAl}_{3}$ at the $\mathrm{Ce} \mathrm{M}_{4,5}$ edges [38], which also gives $| \pm 1 / 2\rangle$ as a ground state. It is to be noted that CEF analysis using the $\mathrm{X}$-ray absorption spectroscopy is not an independent analysis, and it does need information for CEF energy levels from other techniques such as INS study or heat capacity analysis.

Now we discuss the low energy excitations, especially quasielastic linewidths, measured on IN6 with an incident energy $E_{i}=3.1 \mathrm{meV}$ at various temperatures between 2 and $260 \mathrm{~K}$. Figure 14 shows the quasielastic response from $\mathrm{CeAuAl}_{3}$ at various temperatures. It is clear that at $2 \mathrm{~K}$ we have a clear sign of low energy scattering and with increasing temperature the linewidth of the quasielastic line increases with temperature and the quasielastic intensity decreases. The former one gives the estimation of Kondo temperature, while the latter follows the behavior very similar to dc susceptibility. To analyze quantitatively the linewidth and intensity as a function of temperature, we first analyzed the data using a Lorentzian line shape function. Although the fits were very good for the data above $50 \mathrm{~K}$, the data below $50 \mathrm{~K}$, and

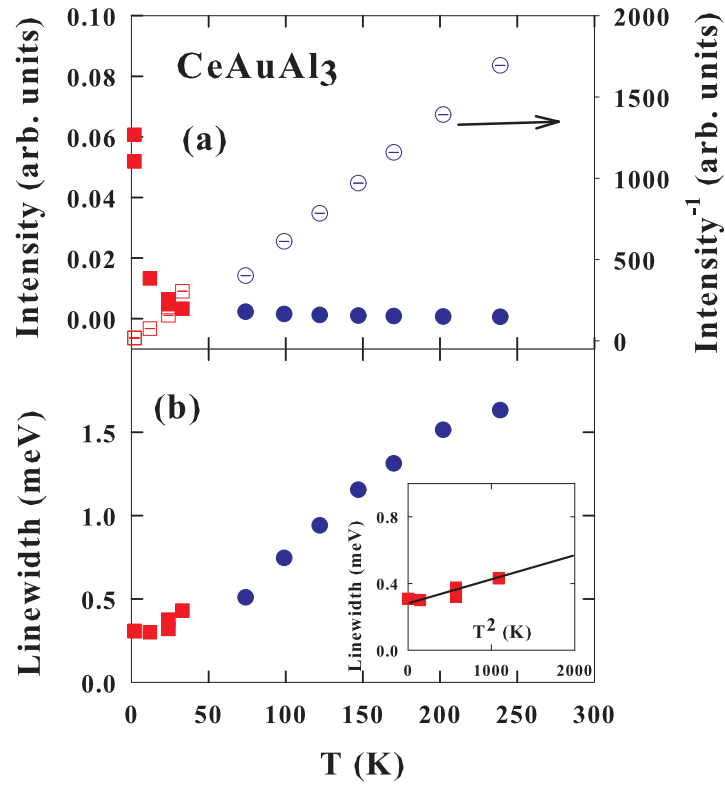

FIG. 15. (Color online) (a) Temperature dependence of the intensity (right $y$ axis inverse intensity) of the quasielastic (QE) excitations and (b) the quasielastic linewidth (solid squares is Gaussian $\sigma \times 2.3548 / 2$ and solid circle Lorentzian (half-width at half-maximum) linewidth estimated from the analysis of low energy inelastic data of IN6 given in Fig. 14 (see text). The inset in (b) shows the low temperature linewidth as a function of $T^{2}\left(\mathrm{~K}^{2}\right)$.

especially at $2 \mathrm{~K}$, were not fitted very well to a Lorentzian line shape. We, therefore, analyzed the low temperature data using a Gaussian line shape function, which showed excellent agreement with the data. The estimated linewidth and the intensity of the quasielastic line are plotted as a function of temperature in Fig. 15. It is interesting to see that the intensity (or inverse intensity) follows CW-type behavior very similar to the dc susceptibility. Furthermore, the linewidth exhibits nearly linear behavior above $50 \mathrm{~K}$, while it shows nearly $T^{2}$ behavior at low temperature [see the inset in Fig. 15(b)]. The value of the linewidth at $2 \mathrm{~K}$ is $\sim 0.3 \mathrm{meV}$, which gives a Kondo temperature of $3.5 \mathrm{~K}$. This value of $T_{\mathrm{K}}$ is in excellent agreement with that estimated from the heat capacity [20]. The observation of a Gaussian line shape below $50 \mathrm{~K}$ suggests that the spin fluctuations are mainly due to intersite spin-spin correlations (there are strong paramagnetic correlations at least up to $30 \mathrm{~K}$ ) rather than single-site spin relaxation observed in many HF systems. This type of a Gaussian line shape and the presence of paramagnetic correlations has been observed in the HF compound YbBiPt [39]. Further, it is to be noted that the quasielastic response of $\mathrm{YbAuCu}_{4}$ and $\mathrm{YbPdCu}_{4}$ also show the presence of two components, Lorentzian and Gaussian, below $10 \mathrm{~K}$ [40]. The observation of the Gaussian component in these compounds has been attributed to a precursor of the magnetic order taking place below $1 \mathrm{~K}$ [40].

\section{CONCLUSIONS}

We have investigated the $\mathrm{HF}$ AFM compound $\mathrm{CeAuAl}_{3}$ using $\mu$ SR and neutron scattering measurements, in addition to magnetization, transport and heat capacity studies. $\mathrm{CeAuAl}_{3}$ 
shows a magnetic phase transition at $1.1 \mathrm{~K}$, with possibly another transition near $0.18 \mathrm{~K}$, as indicated in the ac susceptibility data. The transition at $1.1 \mathrm{~K}$ is a paramagnetic to AFM phase transition that has been clearly seen in the temperature dependence of the $\mu \mathrm{SR}$ initial asymmetry and the relaxation rate. The nature of the second transition at $0.18 \mathrm{~K}$ in the ac susceptibility needs further investigation. Neutron diffraction shows that below $1.1 \mathrm{~K} \mathrm{CeAuAl}_{3}$ exhibits a helical magnetic structure with a propagation vector $\mathbf{k}=$ $(0,0,0.52(1))$ incommensurate with the tetragonal unit cell. Ce moments are ferromagnetically aligned in the $a b$ plane and rotate by an angle $\varphi=93.6^{\circ}$ between neighboring planes in the $c$ direction. The INS reveals well-defined two CEF excitations at 5.1 and $24.7 \mathrm{meV}$ at $4.5 \mathrm{~K}$. From the analysis of the INS data, we have obtained the CEF parameters that can describe the single-crystal susceptibility with the anisotropic molecular field parameters. Our low energy INS study shows a well-defined quasielastic line, which gives $T_{\mathrm{K}}=3.5 \mathrm{~K}$ (in good agreement with $T_{\mathrm{K}}=4 \mathrm{~K}$ estimated from the heat capacity) and further shows evidence of slowdown of spin fluctuations below $30 \mathrm{~K}$, which is well above the magnetic ordering temperature. It is interesting to mention that the INS spectra of $\mathrm{CeAuAl}_{3}$ do not show any sign of CEF-phonon coupling, unlike that observed in $\mathrm{CeCuAl}_{3}$. Further, the overall CEF splitting of $25 \mathrm{meV}$ observed in $\mathrm{CeAuAl}_{3}$ is higher than the overall CEF splitting of $\sim 20 \mathrm{meV}$ observed in $\mathrm{CeCuAl}_{3}$ [23] and $\mathrm{CeRhGe}_{3}$ [10].

Finally, we would like to mention that most of the known HF compounds scale quite well with the Kadowaki-Woods ratio $\left.(\mathrm{KWR}), \mathrm{KWR}=\mathrm{A} / \gamma_{\text {elec }}^{2}\right)$, i.e., a ratio between the coefficient $A$ of the $T^{2}$ variation of the resistivity and the square of electronic contribution $\left(\gamma_{\text {elec }}^{2}\right)$ to $C_{p}$. The KWR is considered universal and has the value of $\sim 10^{-5} \mu \Omega \mathrm{cm}(\mathrm{mole}-\mathrm{K} / \mathrm{mJ})^{2}$ [41-47]. The KWR could be constant under an applied magnetic field whenever the system is far away from a QCP as initially expected. According to the Fermi liquid theory, KWR is proportional to a constant coupling of quasiparticles under exchange interaction, $\alpha_{0}$, and proportional to a parameter that characterizes the shape of the Fermi surface (SFS) [42]. Therefore, the product of these two factors support the universal character of KWR in HF $[42,47]$. In our case, KWR = $9.410^{-5} \mu \Omega \mathrm{cm}(\text { mole }-\mathrm{K} / \mathrm{mJ})^{2}$, which is slightly enhanced with respect to most of known AFM HFs [42-47], and it is quite unaffected by the existence of applied magnetic fields, at least up to $7 \mathrm{~T}$. Then, (1) $\mathrm{CeAuAl}_{3}$ would not be so close to the QPC as initially expected at the beginning, and (2) the SFS is the most likely factor to explain the enhancement of KWR, as the quasiparticle interaction $\alpha_{0}$ hardly changes in most of known HF systems. On the other hand, Wilson ratio (WR) [48-50], which is also used to characterize HF compounds can provide insight into the types of interactions present. The WR depends on two important contributions: (1) the electronic contribution to heat capacity, $\gamma_{\text {elec }}$ and (2) the static magnetic susceptibility, $\chi_{0}$. Both are proportional to the DOS at the Fermi energy, and so they should have similar changes when a magnetic field is applied [47]. In our $\mathrm{CeAuAl}_{3}$ system, WR $\cong 1.6$, which is close to the theoretical value $\mathrm{WR}=1.5$ and also close to 1.46 observed in $\mathrm{CeRu}_{2} \mathrm{Si}_{2}$ [47]. For most of strongly correlated systems, WR $>1$ where the spin fluctuations are enhanced, while charge fluctuations are suppressed.

\section{ACKNOWLEDGMENTS}

We acknowledge Dr. A. Arauzo and Dr. M. Concepción for technical support during the PPMS and x-ray measurements, respectively, and Dr. C. Ritter and Dr. P. Manuel for a valuable discussion on the ND data analysis. We acknowledge Dr. A. Murani, A. Severing, B. D. Rainford, K. A. McEwen, A. M. Strydom, and Dr. V. K. Anand for stimulating discussions on the CEF analysis. We are grateful for funding by the Centre for Materials Physics and chemistry-Science and technology facilities council (CPMC-STFC) (Grant No. CMPC-09108), EU-FEDER (Grants No. MAT2009-10040, No. MAT201231309, and No. DGA-E81) and Fondo Social Europeo.
[1] S. Doniach, Phys. B 91, 231 (1977).

[2] G. R. Stewart, Rev. Mod. Phys. 73, 797 (2001).

[3] Q. Si, S. Rabello, K. Ingersent, and J. L. Smith, Nature (London) 413, 804 (2001).

[4] J. Custers, P. Gegenwart, H. Wilhelm, K. Neumaier, Y. Tokiwa, O. Trovarelli, C. Geibel, F. Steglich, C. Pépin, and P. Coleman, Nature (London) 424, 524 (2003).

[5] H. v. Löhneysen, A. Rosch, M. Vojta, and P. Wölfle, Rev. Mod. Phys. 79, 1015 (2007).

[6] P. Coleman and C. Pépi, Phys. B 312-313, 383 (2002).

[7] J. Flouquet, S. Kambe, L. P. Regnault, P. Haen, J. P. Brison, F. Lapierre, and P. Lejay, Phys. B 215, 77 (1995).

[8] T. Fukuhara, K. Mazewa, H. Ohkuni, J. Sakurai, H. Sato, H. Azuma, K. Sygiyama, Y. Onuki, and K. Kindo, J. Phys. Soc. Jpn. 65, 1559 (1996).

[9] C. M. Varma, Z. Nussinov, and W. van Saarloos, Phys. Rep. 361, 267 (2002).

[10] A. D. Hillier, D. T. Adroja, P. Manuel, V. K. Anand, J. W. Taylor, K. A. McEwen, B. D. Rainford, and M. M. Koza, Phys. Rev. B 85, 134405 (2012).
[11] V. K. Anand, D. T. Adroja, A. D. Hillier, W. Kockelmann, A. Fraile, and A. M. Strydom, J. Phys.: Condens. Matter 23, 276001 (2011).

[12] M. Smidman, D. T. Adroja, A. D. Hillier, L. C. Chapon, J. W. Taylor, V. K. Anand, R. P. Singh, M. R. Lees, E. A. Goremychkin, M. M. Koza, V. V. Krishnamurthy, D. M. Paul, and G. Balakrishnan, Phys. Rev. B 88, 134416 (2013).

[13] E. Bauer, N. Pillmayr, E. Gratz, G. Hilscher, D. Gignoux, and D. Schmitt, Z. Phys B 67, 205 (1987).

[14] Y. Oohara, G. Motoyama, T. Nishioka, and M. Kontani, Technical Report of ISSP, Ser. A, No. 3526, 1 (1999).

[15] T. Muranaka and J. Akimitsu, Phys. C 460-462, 688 (2007).

[16] K. Sugiyama, H. Fuke, K. Kindo, S. Shimohata, A. A. Menovsky, J. A. Mydosh, and M. Date, J. Phys. Soc. Jpn. 59, 3331 (1990).

[17] J. J. M. Franse, P. H. Frings, A. de Visser, A. Menovsky T. T. M. Palstra, P. H. Kes, and J. A. Mydosh, Physica B+C 126, 116 (1984).

[18] K. Oda, Kumada, K. Sugiyama, N. Sato, T. Komatsubara, and M. Date, J. Phys. Soc. Jpn. 63, 3115 (1994). 
[19] N. Kimura, K. Ito, K. Saitoh, Y. Umeda, H. Aoki, and T. Terashima, Phys. Rev. Lett. 95, 247004 (2005).

[20] S. Paschen, E. Felder, and H. R. Ott, Eur. Phys. J. B 2, 169 (1998).

[21] S. Mock, C. Pfleiderer, and H. v. Lohneysen, J. Low Temp. Phys. 115, 1 (1999).

[22] P. Vonlanthen, J. L. Gavilano, B. Ambrosini, and H. R. Otta, Eur. Phys. J. B 7, 9 (1999).

[23] D. T. Adroja, A. del Moral, C. de la Fuente, A. Fraile, E. A. Goremyhkin, J. W. Taylor, A. D. Hillier, and F. FernandezAlonso, Phys. Rev. Lett. 108, 216402 (2012).

[24] F. Hulliger, J. Alloys Compd. 218, 255 (1995).

[25] C. Kittel, Introduction to Solid State Physics, 7th ed. (Wiley, New York, 1996).

[26] K. Hanzawa, K. Ohara, and K. Yosida, J. Phys. Soc. Jpn. 66, 3001 (1997).

[27] H. Yashima, H. Mori, N. Sato, and T. Satoh, J. Magn. Magn. Mater 31-34, 411 (1983).

[28] A. Wasserman, M. Springford, and A. C. Hewson, J. Phys.: Condens. Matter 1, 2669 (1989).

[29] J. W. Rasul, Phys. Rev. B 39, 663 (1989).

[30] R. Kubo and T. Toyabe, in Magnetic Resonance and Relaxation, edited by R. Blinc (North-Holland, Amsterdam, 1996), p. 810.

[31] A. D. Hillier, D. T. Adroja, S. R. Giblin, W. Kockelmann, B. D. Rainford, and S. K. Malik, Phys. Rev. B 76, 174439 (2007).

[32] J. Rodriguez-Carvajal and T. Roisnel, FullProf.98 and WinPLOTR: new Windows 95/NT applications for diffraction commission for powder diffraction, International Union for Crystallography, Newsletter No. 20 (May-August), Summer (1998).
[33] A. S. Wills, Phys. B 276, 680 (2000); Z. Kristallogr. Suppl. 30, 39 (2009).

[34] E. Balcar and S. W. Lovesey, Theory of Magnetic Neutron and Photon Scattering (Clarendon, Oxford, England, 1989).

[35] M. T. Hutchings, Solid State Phys. 16, 227 (1964).

[36] J. Jensen and A. R. Mackintosh, Rare Earth Magnetism: Structures and Excitations (Oxford University Press, Oxford, England, 1991).

[37] H. Sugawara, S. R. Saha, T. D. Matsuda, Y. Aoki, H. Sato, J. L. Gavilano, and H. R. Ott, Phys. B 259, 16 (1999).

[38] A. Severing (private communication).

[39] R. A. Robinson, M. Kohgi, T. Osakabe, P. C. Canfield, T. Kamiyama, T. Nakane, Z. Fisk, and J. D. Thompson, National Laboratory for High Energy Physics, KEK Reports, 92-132, November (1992).

[40] A. Severing, A. P. Murani, J. D. Thompson, Z. Fisk, and C.-K. Loong, Phys. Rev. B 41, 1739 (1990).

[41] K. Kadowaky and S. B. Woods, Solid State Commun. 59, 507 (1986).

[42] O. Tayuka, J. Phys. Soc. Jpn. 67, 4178 (1998).

[43] M. J. Rice, Phys. Rev. Lett. 20, 1439 (1968).

[44] K. Miyake, T. Matsuura, and C. M. Varma, Solid State Commun. 71, 1149 (1989).

[45] N. Tsujii, H. Kontani, and K. Yoshimura, Phys. Rev. Lett. 94, 057201 (2005).

[46] H. Kontani, J. Phys. Soc. Jpn. 73, 515 (2004).

[47] M. A. Continentino, Eur. Phys. J. B 13, 31 (2000).

[48] K. Wilson, Rev. Mod. Phys. 47, 773 (1975).

[49] A. C. Hewson, The Kondo Problem to Heavy Fermions (Cambridge University Press, Cambridge, England, 1993).

[50] Z. Fisk, H. R. Ott, and G. Aeppli, J. Appl. Phys. 26, Suppl. 26-3, 1882 (1987). 